\title{
Geocoding of German Administrative Data
}

\section{vom Berge, Philipp ${ }^{a}$ and Wurdack, Anja ${ }^{a}$}

${ }^{a}$ Research Data Centre (FDZ) of the German Federal Employment Agency (BA) at the Institute for Employment Research (IAB), Germany

\begin{abstract}
Wherever we choose to move, we often find ourselves living close to people with similar social status, income or ethnicity. While many mechanisms have already been proposed to explain the driving factors of segregation, including the willingness to pay for local amenities, a preference for ethnical homogeneity, or the spatial distribution of jobs there is still missing an unified empirical framework to assess the relative importance of these mechanisms. Improvements in urban research requires data at a very granular spatial resoultion. We use geo-tagged administrative micro data to obtain more insights into urban research and develop an empirical framework to measure urban segregation. Our analysis is based on the Integrated Employment Biographies (IEB) from the Institute of Employment Research (IAB). The data excerpt used here is restricted to all main records effective on June 30th 2009. We identified the primary notification for the observation period and restrict our analysis to this. This subset of data has been linked to geocoded address material from the Federal Agency for Cartography and Geodesy (GAB) by a deterministic linkage. The individual data have been aggregated into grid cells with an edge length of 500 meters. The median daily wage has been calculated within each grid cell as well as the percentage of employees earning below several low-earning thresholds, including 2/3 of the national median gross daily wage, 2/3 of the city-specific median gross daily wage. The data from grid cells were used to produce various city-wide segregation indexes. This allows consistent comparisons of segregation in a larger cross-section of cities for the first time. We visually demonstrate the potential of our approach comparing segregation patterns in the the three largest cities in Germany. We find substantial variation across cities in both the spatial pattern of sorting and the extent of separation between social groups. This variation can be used to analyze social segregation and its relations to the local economical and demographical characteristics as well as to help city governments to reduce inequality.
\end{abstract}

Keywords: Geocoding; labour market; segregation; urban planning 